\title{
Effect of Pulse Treatment on Post Harvest Quality of Gerbera (Gerbera jamesonii Bolus ex Hooker) cv. Black Bird
}

\author{
Lyang B. Sangma and A. K. Chaurasiya* \\ Department of Horticulture, NEHU, Tura Campus, Meghalaya, India \\ *Corresponding author
}

\section{Keywords}

Relative fresh weight, Water uptake, Water loss, Gerbera flowers

\section{Article Info}

Accepted: 12 November 2019 Available Online: 10 December 2019

\section{A B S T R A C T}

The experiment was carried out in the department of horticulture NEHU Tura Campus, Meghalaya during the year 2013-2015 by keeping in view of 11 treatments on the effect of postharvest quality of cut Gerbera flower for maintaining the vase life at room temperature. The cut flowers were harvested in the morning and were kept in vase solutions containing gibberrelic acid and benzyl adenine (with distilled water). Sucrose at $4 \%$ was added to all treatments as a base solution. The changes in relative fresh weight, water uptake, water loss, acceptability, scape bending, vase life at $50 \%$ fading and biochemical changes were estimated during the vase period. The results were observed that the relative fresh weight, water uptake, water loss and vase life decreased significantly during the experiment period for all the treatments. During the vase period, gibberrelic acid treatments maintained significantly more favourable relative fresh weight, water uptake, water loss, as compared to control treatment and benzyl adenine solution. The highest values of measured parameters were found more significant with application of gibberrelic acid at $50 \mathrm{ppm}$ solution. The result revealed that the quality attributes and vase life of cut gerbera flowers were improved by the use of gibberrelic acid treatment.

\section{Introduction}

Gerbera is a genus of ornamental plants from the sunflower family (Asteraceae). It is a diploid species with the somatic chromosome number $2 n=50$. It was named in honour of the German botanist and naturalist Traugott Gerber (1743). It has approximately 30 species in the wild, extending to South America, Africa and Tropical Asia. The first scientific description of a Gerbera was made by J.D. Hooker in Curtis's Botanical Magazine in 1889 when he described Gerbera jamesonii, a South African species also known as Transvaal daisy or Barberton Daisy. Gerbera is also commonly known as the African Daisy. India is bestowed with several agro-climatic zones conducive for production of sensitive and delicate flower products. During the decade after liberalization floriculture industries took giant steps in the export arena. This era has seen a dynamic shift from 
sustenance production to commercial production.

According to National Horticulture Database2016-17, the area covered under floriculture in India was 9,43,600 hectares with a production of 3.277 million tonnes. Gerbera flowers are extensively grown in Assam, Meghalaya, Arunachal Pradesh, Nagaland, Tripura, Sikkim, and Manipur covering an area of255.79 lakh ha.

The vase life of gerberas can vary significantly depending upon the postharvest application and degree of sanitation provided. Petal wilt, flower stem bending (commonly called "bent neck") and disease, such as Botrytis, are commonly the basis of reduced vase life with the Gerbera flower.

Use of plant growth regulators such as Gibberellic acid or Benzyl adenine, maintains the flower quality by controlling of microbial growth effectively, reducing the optical density of vase solution, recording lowered scape bending curvature and percentages of bent and wilted flower scapes thereby, increasing the vase life for longer duration as compared to control.

Gibberellic acid is a very potent hormone whose natural occurrence in plants controls their development. Since $\mathrm{GA}_{3}$ regulates growth, applications of very low concentrations can have a profound effect.

Benzyl adenine or BAP is a first-generation synthetic cytokinin that elicits plant growth and development responses. It is an inhibitor of respiratory kinase in plants, and increases post-harvest life of green vegetables.

\section{Materials and Methods}

The experiment was laid down in the Laboratory of Department of Horticulture,
North Eastern Hill University, Tura Campus, Meghalaya, India during the year 2013-2015 with the present research entitled "Effect of pulse treatment on post harvest quality of gerbera (Gerbera jamesonii bolus ex hooker) cv. Black Bird". Tura comes under the sub tropical climate of Meghalaya with the average annual temperature of maximum $31.7^{\circ} \mathrm{C}$ and minimum $10.5^{\circ} \mathrm{C}$, average rainfall $(12.5 \mathrm{~mm}-300 \mathrm{~mm})$ and Relative humidity $(62.5 \%)$.

The eleven different treatments with three replications and the statistically Completely Randomized Block design (CRD) were analysed for the vase life of cut gerbera flower namely $\mathrm{T}_{1}$ - Control (Distilled Water), $\mathrm{T}_{2}-(50$ ppm $\left.\mathrm{GA}_{3}\right), \mathrm{T}_{3}-\left(100 \mathrm{ppm} \mathrm{GA}_{3}\right), \mathrm{T}_{4}-(150 \mathrm{ppm}$ $\left.\mathrm{GA}_{3}\right), \mathrm{T}_{5} \quad-\left(200 \quad \mathrm{ppm} \quad \mathrm{GA}_{3}\right), \mathrm{T}_{6}-(250 \mathrm{ppm}$ $\left.\mathrm{GA}_{3}\right) \mathrm{T}_{7^{-}}$(25 ppm BA-Benzyl Adenine), $\mathrm{T}_{8}-$ (50 ppm BA-Benzyl Adenine), $\mathrm{T}_{9}-(75 \mathrm{ppm}$ BA-Benzyl Adenine $), \mathrm{T}_{10}-(100 \mathrm{ppm} \mathrm{BA}-$ Benzyl Adenine $)$ andT $\mathrm{T}_{11}-(150 \mathrm{ppm}$ BABenzyl Adenine) with 4\% sucrose for the vase solution by adding citric acid for maintaining the $\mathrm{pH}(3.0)$.

The gerbera flowers cv. Black Bird were procured from the District Horti-hub, Rongram, Meghalaya for the experiment. The flowers were kept at ambient temperature and light condition at the experimental site for evaluating the vase life under different concentration of vase solution prepared with gibberrelic acid and benzyl adenine. The climatic parameters like temperature $\left({ }^{\circ} \mathrm{C}\right)$ and relative humidity (\%) recorded during experiment had been furnished in Table 1. The physico-biochemical parameters were analysed by standard method viz. sugars by Lane and Eynon (1923), anthocyanin by Ranganna (2004) and acceptability of flower rated with a team of panelist included colour, texture and bending of the flowers on 5 Point Hedonic Scale by Amerine et al., (1965). 


\section{Results and Discussion}

The physico-chemical observations of fresh cut gerbera $c v$. Black bird was measured with fresh weight $(25.9 \mathrm{~g})$, flower diameter $(78.62 \mathrm{~mm})$ and stalk diameter $(6.90 \mathrm{~mm})$ whereas the total sugar content of flower, reducing sugar, non-reducing and anthocyanin are $2.17 \%, 1.81 \%, 0.35 \%$ and $21.82 / 100 \mathrm{mg}$ respectively.

\section{Study on changes of quality of cut gerbera flower during vase life}

\section{Fresh weight changes}

The relative fresh weight changes (Table 2a) revealed that significant increase in relative fresh weight changes upto 6 days during vase life. Benzyl adenine at $50 \mathrm{ppm}\left(\mathrm{T}_{8}\right)$ showed highest fresh weight $(1.71 \mathrm{~g})$ followed by $\operatorname{control}(2.03 \mathrm{~g})$ and gibberrelic acid at 50 $\operatorname{ppm}(2.04 \mathrm{~g})$ respectively on 6 days. These results are corroborate with the findings of Alaeyet al., 2011 in cut rose flowers. The decrease in relative fresh weight of cut flowers after 6 days could be due to the decrease in water uptake (Bieleski and Reid, 1992; Sereket al., 1995). However, decrease in relative fresh weight changes were observed in all the treatments after 10 days. The decreasing trend in relative fresh weight changes among the treatment combinations irrespective of gibberrelic acid and benzyl adenine may be due to least water uptake and occurrence of scape bending. It was also noticed that lower doses of gibberrelic acid and benzyl adenine predominantly influenced the relative fresh weight changes of gerbera cut flowers as compared to higher doses during advancement of the days under vase solution.

\section{Water uptake}

It was observed that the water uptake by cut flower decreased during the vase life but the maximum water uptake was observed in $\mathrm{T}_{7}$ on the $2^{\text {nd }}$ day $\left(7.34 \mathrm{ml} /\right.$ flower) and $\mathrm{T}_{11}$ on $6^{\text {th }}$ day (18.42 $\mathrm{ml} /$ flower), whereas $\mathrm{T}_{8}$ on $4^{\text {th }}$ day (13.75 ml/flower), $\mathrm{T}_{4}$ on $8^{\text {th }}$ day $(25.17 \mathrm{ml} /$ flower), $\mathrm{T}_{5}$ on $10^{\text {th }}$ day (30.15 ml/flower), $\mathrm{T}_{4}$ on $12^{\text {th }}$ day (35.31 ml/flower), $\mathrm{T}_{1}$ on $14^{\text {th }}$ day (38.03 $\mathrm{ml} /$ flower), $\mathrm{T}_{3}$ on $16^{\text {th }}$ day (44.82 $\mathrm{ml} /$ flower) and $\mathrm{T}_{2}$ on $18^{\text {th }}$ day (51.58 $\mathrm{ml} /$ flower). The lower concentration of gibberelic acid in the vase solution increased the water uptake in the entire study (Table $2 b$ ). It might be due to the fact that gibberelic acid present in the holding solution inhibiting microbial population and reduced the blockage of vascular tissues. The stems of gerbera were highly prone to water stress and the blockage of the base stem results in decreased of water uptake. A very high level of turgidity is necessary for continuation of normal metabolic activities in the cut flowers and sucrose helps in water balance and turgidity. Hence, addition of sucrose to holding solution might have lead to the increased uptake of the holding solution. This was in conformity with the findings of Rogers 1973. The present investigation revealed that the best holding solution for cut gerbera blooms would be a combination of gibberelic acid and sucrose. Observation on fresh weight and water uptake indicated that chemical treatments acted primarily by improving water uptake and consequently extending the vase life.

\section{Water loss}

The water loss increased significantly during experiment, the water loss levels at the initial day were higher than the end of the vase life as shown in Table 2c. The lowest levels of water loss was observed for $\mathrm{T}_{2}, \mathrm{~T}_{8}$ and $\mathrm{T}_{10}$ on $2^{\text {nd }}$ day ( $8.08 \mathrm{ml} /$ flower) during vase period followed by $\mathrm{T}_{3}$ on $4^{\text {th }}$ day $(13.16 \mathrm{ml} /$ flower and $\mathrm{T}_{1}$ on $6^{\text {th }}$ day $(18.32 \mathrm{ml} /$ flower $)$ while the highest was in $\mathrm{T}_{4}$ on $2^{\text {nd }}$ day (9.32 $\mathrm{ml} /$ flower), 
$\mathrm{T}_{8}$ on $4^{\text {th }}$ day $(15.25 \mathrm{ml} /$ flower $)$ and $\mathrm{T}_{4}$ on $6^{\text {th }}$ day $(20.97 \mathrm{ml} /$ flower) respectively.

With respect to the results, the application of gibberelic acid was found more effective than the post-harvest benzyl adenine application in reducing the water loss of cut gerbera flowers after harvest. Results were in agreement with Lu et al., (2010) who reported that the water loss of cut rose flowers increased significantly after harvest.

\section{Acceptability}

Flower acceptability judged by the team of panelist with 5 point hedonic scale included colour and healthiness appearance of cut gerbera flower that were kept in vase solution (Table 3). The appearance of vase life of cut gerbera showed the fastest deterioration of flower colour occurred in $\mathrm{T}_{7}\left(2.20\right.$ score on $8^{\text {th }}$ day in vase). The maximum acceptability of flower colour was obtained in $\mathrm{T}_{2}$ (1.32) and $\mathrm{T}_{3}$ (1.94). The 4 percent vase solution of sucrose with gibberrelic acid at $50 \mathrm{ppm}$ and 100 ppm maintained colours with acceptable colours even after 12 days of the vase life. The deterioration of acceptability of cut gerbera might be due to the microbial activity in vase solution, blockage of turgidity and scape bending.

\section{Degree of scape bending}

The treatment screening for cut gerbera showed that postharvest stem break differs from one treatment to another (Table 4). It was observed the stem bending percentage of 11 treatments is based on curvature angle in 5 different rates. The results were found that $100 \%$ of $\mathrm{T}_{1}, \mathrm{~T}_{2}$ and $\mathrm{T}_{3}$ treatments that the stems are without bending or bending less than $15^{\circ}$; treatments that are resistant to stem break till the $12^{\text {th }}$ day. While $\mathrm{T}_{7}, \mathrm{~T}_{8}, \mathrm{~T}_{9}, \mathrm{~T}_{10}$ and $\mathrm{T}_{11}$ obtained rate $4\left(65^{0}-90^{0}\right)$, that is, bending more than $90^{\circ}$ on the $8^{\text {th }}$ day; treatment that were sensitive to stem break. The scape bending of cut gerbera had a several factor viz. stop the pulsing, infected vase solution and so on.

\section{Sugars}

The sugar content of cut gerbera flowers are shown in Table 6(a). The initial day of total sugar and reducing sugar were found to be highest in the treatment $\mathrm{T}_{10}$ (TS- $2.54 \%$ and RS- $2.15 \%$ ) except non-reducing sugar (NRS$0.56 \%) \mathrm{T}_{11}$ followed by $\mathrm{T}_{4}$ (TS- $2.33 \%$ and RS- $2.02 \%$ ) respectively. It was found that continuous decreasing in trend with increasing vase life of cut gerbera in all the treatments might be due to sugars used in respiration activity and produce the energy for the flowers.

\section{Anthocyanin}

Anthocyanin content of cut gerbera flowers decreased significantly with increased vase life of flower Table 6(b). Maximum anthocyanin content $(24.87 \mathrm{mg} / 100 \mathrm{~g})$ was recorded in $\mathrm{T}_{4}$ followed by $\mathrm{T}_{11}$ $(24.50 \mathrm{mg} / 100 \mathrm{~g})$ on initial day of vase life. However, flowers kept in treatment $\mathrm{T}_{5}$ showed a minimum anthocyanin content (17.75 $\mathrm{mg} / 100 \mathrm{~g}$ ) in the same day of vase life. While, the maximum anthocyanin retention was found in $\mathrm{T}_{4}(23.8 \mathrm{mg} / 100 \mathrm{~g})$ on $8^{\text {th }}$ days of vase life and $\mathrm{T}_{3}(20.61 \mathrm{mg} / 100 \mathrm{~g})$ on $18^{\text {th }}$ day of vase life. Simultaneously, the minimum retention of anthocyanin was in $\mathrm{T}_{5}(15.65$ $\mathrm{mg} / 100 \mathrm{~g})$ on $8^{\text {th }}$ day and $\mathrm{T}_{1}$ (control) -13.45 $\mathrm{mg} / 100 \mathrm{~g}$ on $18^{\text {th }}$ day of vase life. The anthocyanin pigment content in flower cells are stable in low $\mathrm{pH}$, however degradation of anthocyanin are adversely affected by higher $\mathrm{pH}$ in medium and are light sensitive because it is a water soluble pigment. 
Table.1 Meteorological data pertaining to the period of experimentations

\begin{tabular}{|c|c|c|c|c|}
\hline \multirow[t]{2}{*}{ Date } & \multicolumn{4}{|c|}{ Average temperature in ${ }^{0} \mathrm{C}$ and $\mathrm{RH}(\%)$ Daily } \\
\hline & Max & Min & Avg & R.H \\
\hline 01.4 .15 & 32.00 & 20.00 & 26.00 & 94.00 \\
\hline 02.4 .15 & 33.00 & 20.00 & 26.50 & 88.00 \\
\hline 03.4.15 & 33.00 & 21.00 & 27.00 & 90.00 \\
\hline 04.4 .15 & 31.00 & 18.00 & 24.50 & 70.00 \\
\hline 05.4 .15 & 30.00 & 18.00 & 24.00 & 68.00 \\
\hline 06.4.15 & 30.00 & 18.00 & 24.00 & 58.00 \\
\hline 07.4.15 & 31.00 & 19.00 & 25.00 & 62.00 \\
\hline 08.4 .15 & 34.00 & 20.00 & 27.00 & 92.00 \\
\hline 09.4.15 & 34.00 & 20.00 & 27.00 & 94.00 \\
\hline 10.4.15 & 33.00 & 20.00 & 26.50 & 92.00 \\
\hline 11.4.15 & 33.00 & 20.00 & 26.50 & 88.00 \\
\hline 12.4 .15 & 33.00 & 21.00 & 27.00 & 90.00 \\
\hline 13.4.15 & 31.00 & 18.00 & 24.50 & 70.00 \\
\hline 14.4.15 & 30.00 & 18.00 & 24.00 & 68.00 \\
\hline 15.4.15 & 30.00 & 18.00 & 24.00 & 58.00 \\
\hline 16.4.15 & 31.00 & 19.00 & 25.00 & 62.00 \\
\hline 17.4.15 & 30.00 & 18.00 & 24.00 & 68.00 \\
\hline 18.4.15 & 32.00 & 22.00 & 27.00 & 64.00 \\
\hline 19.4.15 & 32.00 & 21.00 & 26.50 & 96.00 \\
\hline 20.4.15 & 30.00 & 21.00 & 25.50 & 93.00 \\
\hline 21.4.15 & 32.00 & 21.00 & 26.50 & 95.00 \\
\hline 22.4 .15 & 32.00 & 22.00 & 27.00 & 96.00 \\
\hline 23.4.15 & 31.00 & 19.00 & 25.00 & 64.00 \\
\hline 24.4.15 & 31.00 & 19.00 & 25.00 & 66.00 \\
\hline 25.4.15 & 30.00 & 18.00 & 24.00 & 92.00 \\
\hline 26.4.15 & 31.00 & 18.00 & 24.50 & 86.00 \\
\hline 27.4.15 & 31.00 & 18.00 & 24.50 & 88.00 \\
\hline 28.4.15 & 30.00 & 18.00 & 24.00 & 88.00 \\
\hline 29.4.15 & 32.00 & 21.00 & 26.50 & 92.00 \\
\hline 30.4 .15 & 29.00 & 20.00 & 24.50 & 89.00 \\
\hline
\end{tabular}


Int.J.Curr.Microbiol.App.Sci (2019) 8(12): 1380-1392

Table.2 Changes in quality parameter of treated cut gerbera flower during vase life

Table.2a Fresh weight changes at two days interval (g)

\begin{tabular}{|c|c|c|c|c|c|c|c|c|c|c|}
\hline \multirow{2}{*}{$\begin{array}{c}\text { Treatmen } \\
\mathbf{t}\end{array}$} & 0 & 2 & 4 & 6 & 8 & 10 & 12 & 14 & 16 & 18 \\
\hline $\mathbf{T}_{\mathbf{1}}$ & 27.10 & 27.90 & 28.40 & 29.13 & 30.49 & 28.98 & 26.31 & 23.69 & 21.82 & 19.65 \\
\hline $\mathbf{T}_{\mathbf{2}}$ & 26.46 & 27.53 & 28.10 & 28.50 & 29.20 & 28.59 & 26.37 & 24.75 & 23.45 & 21.22 \\
\hline $\mathbf{T}_{\mathbf{3}}$ & 25.34 & 26.41 & 26.98 & 27.04 & 28.08 & 27.86 & 26.00 & 24.61 & 23.01 & 20.85 \\
\hline $\mathbf{T}_{\mathbf{4}}$ & 25.84 & 26.90 & 27.14 & 28.26 & 28.24 & 27.21 & 25.76 & 23.80 & 0.00 & 0.00 \\
\hline $\mathbf{T}_{\mathbf{5}}$ & 24.41 & 25.47 & 26.04 & 27.44 & 27.81 & 26.78 & 25.24 & 23.68 & 0.00 & 0.00 \\
\hline $\mathbf{T}_{\mathbf{6}}$ & 24.09 & 25.15 & 26.06 & 27.12 & 28.49 & 27.21 & 25.82 & 24.35 & 0.00 & 0.00 \\
\hline $\mathbf{T}_{\mathbf{7}}$ & 25.50 & 26.56 & 26.80 & 27.20 & 0.00 & 0.00 & 0.00 & 0.00 & 0.00 & 0.00 \\
\hline $\mathbf{T}_{\mathbf{8}}$ & 27.48 & 28.55 & 28.82 & 29.18 & 0.00 & 0.00 & 0.00 & 0.00 & 0.00 & 0.00 \\
\hline $\mathbf{T}_{\mathbf{9}}$ & 25.75 & 26.91 & 27.88 & 27.45 & 0.00 & 0.00 & 0.00 & 0.00 & 0.00 & 0.00 \\
\hline $\mathbf{T}_{\mathbf{1 0}}$ & 26.40 & 27.46 & 27.70 & 28.10 & 0.00 & 0.00 & 0.00 & 0.00 & 0.00 & 0.00 \\
\hline $\mathbf{T}_{\mathbf{1 1}}$ & 25.33 & 26.40 & 26.96 & 27.03 & 0.00 & 0.00 & 0.00 & 0.00 & 0.00 & 0.00 \\
\hline $\mathbf{C D}(\mathbf{0 . 0 5})$ & 0.79 & 1.07 & 0.94 & 1.14 & N/A & N/A & N/A & N/A & N/A & N/A \\
\hline $\mathbf{C V}(\mathbf{\%})$ & 1.81 & 2.36 & 2.04 & 2.41 & & & & & & \\
\hline
\end{tabular}

N/A- Not applicable due to senescence and mortality of some treatments

Table.2b Water uptake at two days interval (ml)

\begin{tabular}{|c|c|c|c|c|c|c|c|c|c|}
\hline Treatment & \multicolumn{90}{|c|}{ Days } \\
\cline { 2 - 11 } & 2 & 4 & 6 & 8 & 10 & 12 & 14 & 16 & 18 \\
\hline $\mathbf{T}_{\mathbf{1}}$ & 6.81 & 12.38 & 16.90 & 24.40 & 28.57 & 33.41 & 38.03 & 43.46 & 47.83 \\
\hline $\mathbf{T}_{\mathbf{2}}$ & 6.09 & 12.35 & 18.01 & 23.28 & 28.91 & 32.64 & 36.90 & 44.42 & 51.58 \\
\hline $\mathbf{T}_{\mathbf{3}}$ & 6.15 & 11.82 & 17.38 & 24.90 & 29.12 & 33.31 & 37.32 & 44.82 & 49.60 \\
\hline $\mathbf{T}_{\mathbf{4}}$ & 7.30 & 11.93 & 17.93 & 25.17 & 29.79 & 35.31 & 35.17 & 0 & 0 \\
\hline $\mathbf{T}_{\mathbf{5}}$ & 7.18 & 13.12 & 17.38 & 25.04 & 30.15 & 34.76 & 35.34 & 0 & 0 \\
\hline $\mathbf{T}_{\mathbf{6}}$ & 6.66 & 12.45 & 16.96 & 24.60 & 29.85 & 33.61 & 37.07 & 0 & 0 \\
\hline $\mathbf{T}_{\mathbf{7}}$ & 7.34 & 12.55 & 16.74 & 0 & 0 & 0 & 0 & 0 & 0 \\
\hline $\mathbf{T}_{\mathbf{8}}$ & 6.37 & 13.75 & 17.69 & 0 & 0 & 0 & 0 & 0 & 0 \\
\hline $\mathbf{T}_{\mathbf{9}}$ & 6.47 & 12.36 & 17.2 & 0 & 0 & 0 & 0 & 0 & 0 \\
\hline $\mathbf{T}_{\mathbf{1 0}}$ & 6.44 & 13.46 & 17.56 & 0 & 0 & 0 & 0 & 0 & 0 \\
\hline $\mathbf{T}_{\mathbf{1}}$ & 6.03 & 12.72 & 18.42 & 0 & 0 & 0 & 0 & 0 & 0 \\
\hline $\mathbf{C D}(\mathbf{0 . 0 5})$ & 0.76 & 0.9 & 0.87 & N/A & N/A & N/A & N/A & N/A & N/A \\
\hline $\mathbf{C V}(\mathbf{\%})$ & 6.76 & 4.22 & 2.98 & & & & & & \\
\hline
\end{tabular}

N/A- Not applicable due to senescence and mortality of some treatments 
Int.J.Curr.Microbiol.App.Sci (2019) 8(12): 1380-1392

Table.2c Water loss at two days interval (ml)

\begin{tabular}{|c|c|c|c|c|c|c|c|c|c|}
\hline \multirow{2}{*}{ Treatment } & \multicolumn{7}{|c|}{ Days } \\
\cline { 2 - 12 } & 2 & 4 & 6 & 8 & 10 & 12 & 14 & 16 & 18 \\
\hline $\mathbf{T}_{\mathbf{1}}$ & 8.59 & 14.07 & 18.32 & 25.4 & 30.35 & 35.05 & 39.52 & 44.69 & 50.10 \\
\hline $\mathbf{T}_{\mathbf{2}}$ & 8.08 & 14.20 & 18.34 & 25.00 & 30.93 & 33.77 & 38.61 & 46.16 & 52.91 \\
\hline $\mathbf{T}_{\mathbf{3}}$ & 8.16 & 13.16 & 19.31 & 26.31 & 31.10 & 31.89 & 38.94 & 45.89 & 51.15 \\
\hline $\mathbf{T}_{\mathbf{4}}$ & 9.32 & 13.69 & 20.97 & 26.23 & 31.08 & 35.31 & 36.08 & 0.00 & 0.00 \\
\hline $\mathbf{T}_{\mathbf{5}}$ & 9.15 & 14.51 & 19.53 & 26.88 & 31.49 & 35.94 & 36.73 & 0.00 & 0.00 \\
\hline $\mathbf{T}_{\mathbf{6}}$ & 8.45 & 13.96 & 18.34 & 26.77 & 31.57 & 35.38 & 38.13 & 0.00 & 0.00 \\
\hline $\mathbf{T}_{\mathbf{7}}$ & 8.96 & 14.42 & 19.65 & 0.00 & 0.00 & 0.00 & 0.00 & 0.00 & 0.00 \\
\hline $\mathbf{T}_{\mathbf{8}}$ & 8.08 & 15.25 & 19.85 & 0.00 & 0.00 & 0.00 & 0.00 & 0.00 & 0.00 \\
\hline $\mathbf{T}_{\mathbf{9}}$ & 8.15 & 14.4 & 19.67 & 0.00 & 0.00 & 0.00 & 0.00 & 0.00 & 0.00 \\
\hline $\mathbf{T}_{\mathbf{1 0}}$ & 8.08 & 14.83 & 19.56 & 0.00 & 0.00 & 0.00 & 0.00 & 0.00 & 0.00 \\
\hline $\mathbf{T}_{\mathbf{1 1}}$ & 9.10 & 14.35 & 20.03 & 0.00 & 0.00 & 0.00 & 0.00 & 0.00 & 0.00 \\
\hline $\mathbf{C D}(\mathbf{0 . 0 5})$ & 0.92 & 0.91 & 0.99 & N/A & N/A & N/A & N/A & N/A & N/A \\
\hline $\mathbf{C V}(\mathbf{\%})$ & 6.33 & 3.75 & 3.07 & & & & & & \\
\hline
\end{tabular}

N/A- Not applicable due to senescence and mortality of some treatments

Table.3 Changes in quality attributes during vase life of cut gerbera

\begin{tabular}{|c|c|c|c|c|c|c|c|c|c|c|}
\hline \multirow{2}{*}{ Treatment } & \multicolumn{7}{|c|}{ Acceptability of cut gerbera at two days interval by 5 Point Hedonic Scale } \\
\cline { 2 - 15 } & \multicolumn{2}{|c|}{ Days } \\
\cline { 2 - 15 } & 0 & 2 & 4 & 6 & 8 & 10 & 12 & 14 & 16 & 18 \\
\hline $\mathbf{T}_{\mathbf{1}}$ & 4.94 & 4.64 & 4.26 & 4.12 & 3.80 & 3.66 & 3.14 & 2.6 & 2.28 & 1.32 \\
\hline $\mathbf{T}_{\mathbf{2}}$ & 5.00 & 4.96 & 4.92 & 4.8 & 4.76 & 4.54 & 3.52 & 3.14 & 2.74 & 1.94 \\
\hline $\mathbf{T}_{\mathbf{3}}$ & 5.00 & 4.92 & 4.88 & 4.76 & 4.70 & 4.18 & 3.22 & 2.82 & 2.44 & 1.50 \\
\hline $\mathbf{T}_{\mathbf{4}}$ & 4.94 & 4.60 & 4.28 & 4.08 & 3.86 & 3.68 & 2.80 & 2.62 & 0 & 0 \\
\hline $\mathbf{T}_{\mathbf{5}}$ & 5.00 & 4.66 & 4.28 & 4.04 & 3.90 & 3.80 & 2.52 & 2.42 & 0 & 0 \\
\hline $\mathbf{T}_{\mathbf{6}}$ & 4.96 & 4.62 & 4.28 & 4.04 & 3.84 & 3.70 & 2.70 & 2.34 & 0 & 0 \\
\hline $\mathbf{T}_{\mathbf{7}}$ & 4.98 & 4.62 & 4.32 & 4.00 & 2.20 & 1.60 & 1.02 & 0 & 0 & 0 \\
\hline $\mathbf{T}_{\mathbf{8}}$ & 4.98 & 4.68 & 4.32 & 3.94 & 2.22 & 1.62 & 0.94 & 0 & 0 & 0 \\
\hline $\mathbf{T}_{\mathbf{9}}$ & 4.94 & 4.64 & 4.28 & 3.96 & 2.28 & 1.58 & 1.06 & 0 & 0 & 0 \\
\hline $\mathbf{T}_{\mathbf{1 0}}$ & 5.00 & 4.64 & 4.26 & 3.94 & 2.26 & 1.64 & 0.94 & 0 & 0 & 0 \\
\hline $\mathbf{T}_{\mathbf{1 1}}$ & 4.96 & 4.62 & 4.24 & 3.88 & 2.32 & 1.58 & 0.98 & 0 & 0 & 0 \\
\hline
\end{tabular}

N/A- Not applicable due to senescence and mortality of some treatments 
Table.4 Changes in Scape bending during vase life of cut gerbera (\%)

\begin{tabular}{|c|c|c|c|c|c|}
\hline \multirow{2}{*}{ Treatment } & \multicolumn{5}{|c|}{ Initial Day } \\
\cline { 2 - 6 } & $0-15$ & $15-25$ & $25-65$ & $65-90$ & $>90$ \\
\hline $\mathbf{T}_{\mathbf{1}}$ & 100 & 0 & 0 & 0 & 0 \\
\hline $\mathbf{T}_{\mathbf{2}}$ & 100 & 0 & 0 & 0 & 0 \\
\hline $\mathbf{T}_{\mathbf{3}}$ & 100 & 0 & 0 & 0 & 0 \\
\hline $\mathbf{T}_{\mathbf{4}}$ & 100 & 0 & 0 & 0 & 0 \\
\hline $\mathbf{T}_{\mathbf{5}}$ & 100 & 0 & 0 & 0 & 0 \\
\hline $\mathbf{T}_{\mathbf{6}}$ & 100 & 0 & 0 & 0 & 0 \\
\hline $\mathbf{T}_{\mathbf{7}}$ & 100 & 0 & 0 & 0 & 0 \\
\hline $\mathbf{T}_{\mathbf{8}}$ & 100 & 0 & 0 & 0 & 0 \\
\hline $\mathbf{T}_{\mathbf{9}}$ & 100 & 0 & 0 & 0 & 0 \\
\hline $\mathbf{T}_{\mathbf{1 0}}$ & 100 & 0 & 0 & 0 & 0 \\
\hline $\mathbf{T}_{\mathbf{1 1}}$ & 100 & 0 & 0 & 0 & 0 \\
\hline
\end{tabular}

Table.5 Degree of scape bending at two days interval (\%)

\begin{tabular}{|c|c|c|c|c|c|}
\hline \multirow{2}{*}{ Treatment } & \multicolumn{5}{|c|}{$\mathbf{4}^{\text {th }}$ day } \\
\cline { 2 - 6 } & $0-15$ & $15-25$ & $25-65$ & $65-90$ & $>90$ \\
\hline $\mathbf{T}_{\mathbf{1}}$ & 100 & 0 & 0 & 0 & 0 \\
\hline $\mathbf{T}_{\mathbf{2}}$ & 100 & 0 & 0 & 0 & 0 \\
\hline $\mathbf{T}_{\mathbf{3}}$ & 100 & 0 & 0 & 0 & 0 \\
\hline $\mathbf{T}_{\mathbf{4}}$ & 100 & 0 & 0 & 0 & 0 \\
\hline $\mathbf{T}_{\mathbf{5}}$ & 100 & 0 & 0 & 0 & 0 \\
\hline $\mathbf{T}_{\mathbf{6}}$ & 100 & 0 & 0 & 0 & 0 \\
\hline $\mathbf{T}_{\mathbf{7}}$ & 0 & 0 & 22.22 & 0 & 0 \\
\hline $\mathbf{T}_{\mathbf{8}}$ & 0 & 0 & 22.22 & 0 & 0 \\
\hline $\mathbf{T}_{\mathbf{9}}$ & 0 & 0 & 11.11 & 0 & 0 \\
\hline $\mathbf{T}_{\mathbf{1 0}}$ & 0 & 0 & 11.11 & 0 & 0 \\
\hline $\mathbf{T}_{\mathbf{1 1}}$ & 0 & 0 & 33.33 & 0 & 0 \\
\hline
\end{tabular}

\begin{tabular}{|c|c|c|c|c|c|}
\hline \multirow{2}{*}{ Treatment } & \multicolumn{5}{|c|}{$\mathbf{6}^{\text {th }}$ day } \\
\cline { 2 - 6 } & $0-15$ & $15-25$ & $25-65$ & $65-90$ & $>90$ \\
\hline $\mathbf{T}_{\mathbf{1}}$ & 100 & 0 & 0 & 0 & 0 \\
\hline $\mathbf{T}_{\mathbf{2}}$ & 100 & 0 & 0 & 0 & 0 \\
\hline $\mathbf{T}_{\mathbf{3}}$ & 100 & 0 & 0 & 0 & 0 \\
\hline $\mathbf{T}_{\mathbf{4}}$ & 100 & 0 & 0 & 0 & 0 \\
\hline $\mathbf{T}_{\mathbf{5}}$ & 100 & 0 & 0 & 0 & 0 \\
\hline $\mathbf{T}_{\mathbf{6}}$ & 100 & 0 & 0 & 0 & 0 \\
\hline $\mathbf{T}_{\mathbf{7}}$ & 0 & 0 & 66.66 & 0 & 0 \\
\hline $\mathbf{T}_{\mathbf{8}}$ & 0 & 11.11 & 55.55 & 0 & 0 \\
\hline $\mathbf{T}_{\mathbf{9}}$ & 0 & 0 & 66.66 & 0 & 0 \\
\hline $\mathbf{T}_{\mathbf{1 0}}$ & 0 & 0 & 77.77 & 0 & 0 \\
\hline $\mathbf{T}_{\mathbf{1 1}}$ & 0 & 0 & 77.77 & 0 & 0 \\
\hline
\end{tabular}




\begin{tabular}{|c|c|c|c|c|c|}
\hline \multirow{2}{*}{ Treatment } & \multicolumn{5}{|c|}{$8^{\text {th }}$ day } \\
\cline { 2 - 6 } & \multicolumn{5}{|c|}{ Degree of scape bending } \\
\hline & $0-15$ & $15-25$ & $25-65$ & $65-90$ & $>90$ \\
\hline $\mathbf{T}_{\mathbf{1}}$ & 100 & 0 & 0 & 0 & 0 \\
\hline $\mathbf{T}_{\mathbf{2}}$ & 100 & 0 & 0 & 0 & 0 \\
\hline $\mathbf{T}_{\mathbf{3}}$ & 100 & 0 & 0 & 0 & 0 \\
\hline $\mathbf{T}_{\mathbf{4}}$ & 0 & & 33.33 & 0 & 0 \\
\hline $\mathbf{T}_{\mathbf{5}}$ & 0 & 11.11 & 11.11 & 0 & 0 \\
\hline $\mathbf{T}_{\mathbf{6}}$ & 0 & & 44.44 & 0 & 0 \\
\hline $\mathbf{T}_{\mathbf{7}}$ & 0 & 0 & 0 & 0 & 100 \\
\hline $\mathbf{T}_{\mathbf{8}}$ & 0 & 0 & 0 & 0 & 100 \\
\hline $\mathbf{T}_{\mathbf{9}}$ & 0 & 0 & 0 & 0 & 100 \\
\hline $\mathbf{T}_{\mathbf{1 0}}$ & 0 & 0 & 0 & 0 & 100 \\
\hline $\mathbf{T}_{\mathbf{1 1}}$ & 0 & 0 & 0 & 0 & 100 \\
\hline
\end{tabular}

\begin{tabular}{|c|c|c|c|c|c|}
\hline \multirow{2}{*}{ Treatment } & \multicolumn{5}{|c|}{$\mathbf{1 0}^{\text {th }}$ day } \\
\cline { 2 - 6 } & $0-15$ & $15-25$ & $25-65$ & $65-90$ & $>90$ \\
\hline $\mathbf{T}_{\mathbf{1}}$ & 100 & 0 & 0 & 0 & 0 \\
\hline $\mathbf{T}_{\mathbf{2}}$ & 100 & 0 & 0 & 0 & 0 \\
\hline $\mathbf{T}_{\mathbf{3}}$ & 100 & 0 & 0 & 0 & 0 \\
\hline $\mathbf{T}_{\mathbf{4}}$ & 0 & 0 & 33.33 & 0 & 0 \\
\hline $\mathbf{T}_{\mathbf{5}}$ & 0 & 11.11 & 11.11 & 0 & 0 \\
\hline $\mathbf{T}_{\mathbf{6}}$ & 0 & & 44.44 & 0 & 0 \\
\hline $\mathbf{T}_{\mathbf{7}}$ & 0 & 0 & 0 & 0 & 100 \\
\hline $\mathbf{T}_{\mathbf{8}}$ & 0 & 0 & 0 & 0 & 100 \\
\hline $\mathbf{T}_{\mathbf{9}}$ & 0 & 0 & 0 & 0 & 100 \\
\hline $\mathbf{T}_{\mathbf{1 0}}$ & 0 & 0 & 0 & 0 & 100 \\
\hline $\mathbf{T}_{\mathbf{1 1}}$ & 0 & 0 & 0 & 0 & 100 \\
\hline
\end{tabular}

\begin{tabular}{|c|c|c|c|c|c|}
\hline & \multicolumn{5}{|c|}{$\mathbf{1 2}^{\text {th }}$ day } \\
\hline Treatment & \multicolumn{5}{|c|}{ Degree of scape bending } \\
\hline & $0-15$ & $15-25$ & $25-65$ & $65-90$ & $>90$ \\
\hline $\mathbf{T}_{\mathbf{1}}$ & 0 & 0 & 22.22 & 0 & 0 \\
\hline $\mathbf{T}_{\mathbf{2}}$ & 0 & 11.11 & 22.22 & 0 & 0 \\
\hline $\mathbf{T}_{\mathbf{3}}$ & 0 & 11.11 & 44.44 & 0 & 0 \\
\hline $\mathbf{T}_{\mathbf{4}}$ & 0 & 0 & 33.33 & 0 & 0 \\
\hline $\mathbf{T}_{\mathbf{5}}$ & 0 & 0 & 44.44 & 0 & 0 \\
\hline $\mathbf{T}_{\mathbf{6}}$ & 0 & 0 & 33.33 & 33.33 & 0 \\
\hline $\mathbf{T}_{\mathbf{7}}$ & 0 & 0 & 0 & 0 & 0 \\
\hline $\mathbf{T}_{\mathbf{8}}$ & 0 & 0 & 0 & 0 & 0 \\
\hline $\mathbf{T}_{\mathbf{9}}$ & 0 & 0 & 0 & 0 & 0 \\
\hline $\mathbf{T}_{\mathbf{1 0}}$ & 0 & 0 & 0 & 0 & 0 \\
\hline $\mathbf{T}_{\mathbf{1 1}}$ & 0 & 0 & 0 & 0 & 0 \\
\hline
\end{tabular}




\begin{tabular}{|c|c|c|c|c|c|}
\hline & \multicolumn{5}{|c|}{$14^{\text {th }}$ day } \\
\hline Treatment & \multicolumn{5}{|c|}{ Degree of scape bending } \\
\hline & $0-15$ & $15-25$ & $25-65$ & $65-90$ & $>90$ \\
\hline $\mathbf{T}_{\mathbf{1}}$ & 0 & 0 & 44.44 & 0 & 0 \\
\hline $\mathbf{T}_{\mathbf{2}}$ & 0 & 22.22 & 22.22 & 0 & 0 \\
\hline $\mathbf{T}_{\mathbf{3}}$ & 0 & 11.11 & 44.44 & 0 & 0 \\
\hline $\mathbf{T}_{\mathbf{4}}$ & 0 & 0 & 55.55 & 0 & 0 \\
\hline $\mathbf{T}_{\mathbf{5}}$ & 0 & 0 & 33.33 & 11.11 & 0 \\
\hline $\mathbf{T}_{\mathbf{6}}$ & 0 & 0 & 33.33 & 33.33 & 0 \\
\hline $\mathbf{T}_{\mathbf{7}}$ & 0 & 0 & 0 & 0 & 0 \\
\hline $\mathbf{T}_{\mathbf{8}}$ & 0 & 0 & 0 & 0 & 0 \\
\hline $\mathbf{T}_{\mathbf{9}}$ & 0 & 0 & 0 & 0 & 0 \\
\hline $\mathbf{T}_{\mathbf{1 0}}$ & 0 & 0 & 0 & 0 & 0 \\
\hline $\mathbf{T}_{\mathbf{1 1}}$ & 0 & 0 & 0 & 0 & 0 \\
\hline
\end{tabular}

\begin{tabular}{|c|c|c|c|c|c|}
\hline & \multicolumn{5}{|c|}{$\mathbf{1 6}^{\text {th }}$ day } \\
\hline Treatment & \multicolumn{5}{|c|}{ Degree of scape bending } \\
\hline & $0-15$ & $15-25$ & $25-65$ & $65-90$ & $>90$ \\
\hline $\mathbf{T}_{\mathbf{1}}$ & 0 & 0 & 44.44 & 0 & 0 \\
\hline $\mathbf{T}_{\mathbf{2}}$ & 0 & 11.11 & 33.33 & 0 & 0 \\
\hline $\mathbf{T}_{\mathbf{3}}$ & 0 & 11.11 & 44.44 & 0 & 0 \\
\hline $\mathbf{T}_{\mathbf{4}}$ & 0 & 0 & 0 & 0 & 100 \\
\hline $\mathbf{T}_{\mathbf{5}}$ & 0 & 0 & 0 & 0 & 100 \\
\hline $\mathbf{T}_{\mathbf{6}}$ & 0 & 0 & 0 & 0 & 100 \\
\hline $\mathbf{T}_{\mathbf{7}}$ & 0 & 0 & 0 & 0 & 100 \\
\hline $\mathbf{T}_{\mathbf{8}}$ & 0 & 0 & 0 & 0 & 100 \\
\hline $\mathbf{T}_{\mathbf{9}}$ & 0 & 0 & 0 & 0 & 100 \\
\hline $\mathbf{T}_{\mathbf{1 0}}$ & 0 & 0 & 0 & 0 & 100 \\
\hline $\mathbf{T}_{\mathbf{1 1}}$ & 0 & 0 & 0 & 0 & 100 \\
\hline
\end{tabular}

\begin{tabular}{|c|c|c|c|c|c|}
\hline \multirow{2}{*}{ Treatment } & \multicolumn{5}{|c|}{$\mathbf{1 8}^{\text {th }}$ day } \\
\cline { 2 - 6 } & $0-15$ & $15-25$ & $25-65$ & $65-90$ & $>90$ \\
\hline $\mathbf{T}_{\mathbf{1}}$ & 0 & 0 & 44.44 & 0 & 0 \\
\hline $\mathbf{T}_{\mathbf{2}}$ & 0 & 11.11 & 33.33 & 0 & 0 \\
\hline $\mathbf{T}_{\mathbf{3}}$ & 0 & 11.11 & 44.44 & 0 & 0 \\
\hline $\mathbf{T}_{\mathbf{4}}$ & 0 & 0 & 0 & 0 & 100 \\
\hline $\mathbf{T}_{\mathbf{5}}$ & 0 & 0 & 0 & 0 & 100 \\
\hline $\mathbf{T}_{\mathbf{6}}$ & 0 & 0 & 0 & 0 & 100 \\
\hline $\mathbf{T}_{\mathbf{7}}$ & 0 & 0 & 0 & 0 & 100 \\
\hline $\mathbf{T}_{\mathbf{8}}$ & 0 & 0 & 0 & 0 & 100 \\
\hline $\mathbf{T}_{\mathbf{9}}$ & 0 & 0 & 0 & 0 & 100 \\
\hline $\mathbf{T}_{\mathbf{1 0}}$ & 0 & 0 & 0 & 0 & 100 \\
\hline $\mathbf{T}_{\mathbf{1 1}}$ & 0 & 0 & 0 & 0 & 100 \\
\hline
\end{tabular}


Table.6 Changes in Bio-chemical parameters of cut gerbera flower during vase life

Table.6a Total, Reducing and Non-reducing Sugar

\begin{tabular}{|c|c|c|c|c|c|c|c|c|c|}
\hline \multirow{2}{*}{ Treatment } & \multicolumn{3}{|c|}{ 0 Day } & \multicolumn{3}{c|}{$\mathbf{8}^{\text {th }}$ Day } & \multicolumn{3}{c|}{$\mathbf{1 8}^{\text {th }}$ Day } \\
\cline { 2 - 11 } & TS & RS & NRS & TS & RS & NRS & TS & RS & NRS \\
\hline $\mathbf{T}_{\mathbf{1}}$ & 1.84 & 1.67 & 0.21 & 1.71 & 1.53 & 0.2 & 1.50 & 1.23 & 0.19 \\
\hline $\mathbf{T}_{\mathbf{2}}$ & 2.18 & 1.79 & 0.43 & 2.11 & 1.70 & 0.43 & 1.44 & 1.20 & 0.27 \\
\hline $\mathbf{T}_{\mathbf{3}}$ & 2.00 & 1.70 & 0.34 & 1.95 & 1.63 & 0.34 & 1.39 & 1.16 & 0.25 \\
\hline $\mathbf{T}_{\mathbf{4}}$ & 2.33 & 2.02 & 0.35 & 2.28 & 1.91 & 0.39 & 0 & 0 & 0 \\
\hline $\mathbf{T}_{\mathbf{5}}$ & 2.25 & 1.96 & 0.33 & 2.23 & 1.88 & 0.38 & 0 & 0 & 0 \\
\hline $\mathbf{T}_{\mathbf{6}}$ & 1.98 & 1.63 & 0.39 & 1.93 & 1.54 & 0.41 & 0 & 0 & 0 \\
\hline $\mathbf{T}_{\mathbf{7}}$ & 2.04 & 1.72 & 0.35 & 0 & 0 & 0 & 0 & 0 & 0 \\
\hline $\mathbf{T}_{\mathbf{8}}$ & 2.11 & 1.59 & 0.55 & 0 & 0 & 0 & 0 & 0 & 0 \\
\hline $\mathbf{T}_{\mathbf{9}}$ & 1.94 & 1.63 & 0.35 & 0 & 0 & 0 & 0 & 0 & 0 \\
\hline $\mathbf{T}_{\mathbf{1 0}}$ & 2.54 & 2.15 & 0.43 & 0 & 0 & 0 & 0 & 0 & 0 \\
\hline $\mathbf{T}_{\mathbf{1 1}}$ & 2.28 & 1.76 & 0.56 & 0 & 0 & 0 & 0 & 0 & 0 \\
\hline $\mathbf{C D}(\mathbf{0 . 0 5})$ & 0.16 & 0.08 & 0.08 & N/A & N/A & N/A & N/A & N/A & N/A \\
\hline $\mathbf{C V}(\boldsymbol{\%})$ & 4.52 & 2.81 & 13.13 & & & & & & \\
\hline
\end{tabular}

N/A- Not applicable due to senescence and mortality of some treatments

TS=Total Sugar, RS=Reducing Sugar and NRS=Non-Reducing Sugar

Table.6b Changes in anthocyanin content $(\mathrm{mg} / 100 \mathrm{~g})$ during vase life of cut gerbera

\begin{tabular}{|c|c|c|c|}
\hline \multirow{2}{*}{ Treatment } & \multicolumn{3}{|c|}{ Days } \\
\cline { 2 - 4 } & 0 & 8 & 18 \\
\hline $\mathbf{T}_{\mathbf{1}}$ & 19.19 & 17.59 & 13.45 \\
\hline $\mathbf{T}_{\mathbf{2}}$ & 21.75 & 20.05 & 18.43 \\
\hline $\mathbf{T}_{\mathbf{3}}$ & 24.25 & 22.73 & 20.61 \\
\hline $\mathbf{T}_{\mathbf{4}}$ & 24.87 & 23.29 & 0.00 \\
\hline $\mathbf{T}_{\mathbf{5}}$ & 17.78 & 15.65 & 0.00 \\
\hline $\mathbf{T}_{\mathbf{6}}$ & 22.61 & 20.63 & 0.00 \\
\hline $\mathbf{T}_{\mathbf{7}}$ & 19.61 & 0.00 & 0.00 \\
\hline $\mathbf{T}_{\mathbf{8}}$ & 24.26 & 0.00 & 0.00 \\
\hline $\mathbf{T}_{\mathbf{9}}$ & 17.91 & 0.00 & 0.00 \\
\hline $\mathbf{T}_{\mathbf{1 0}}$ & 23.82 & 0.00 & 0.00 \\
\hline $\mathbf{T}_{\mathbf{1 1}}$ & 24.50 & 0.00 & 0.00 \\
\hline $\mathbf{C D}(\mathbf{0 . 0 5})$ & 1.77 & $\mathrm{~N} / \mathrm{A}$ & N/A \\
\hline $\mathbf{C V}(\mathbf{\%})$ & 4.81 & & \\
\hline
\end{tabular}

N/A- Not applicable due to senescence and mortality of some treatments 
A similar line of work was presented by Zakyet al., (2013) on the effect of vase life of cut gerbera with holding solution.

The short vase life of the cut flowers is one of the most important problems. To enhance the vase life of the cut gerbera, various treatment combinations are recommended. In the present study, influence of gibberelic acid and benzyl adenine on keeping quality and vase life of cut gerbera flowers were investigated. During investigation, it was noticed that cut gerbera in vase solution showed same behaviour in all measured factors for the treatments. The significant increase in relative fresh weight and water uptake enhanced the vase life, while, water loss significantly increased during vase period. In addition, significant differences were observed between control and gibberrelic acid treatment combinations in all measured parameters. In terms of overall performance, application of gibberrelic acid is found to be more effective than benzyl adenine. Gibberelic acid at the rate of $50 \mathrm{ppm}$ was able to increase vase life of cut gerbera flowers by maintaining the plant-water relationship. Thus, the present investigation concluded that gibberrelic acid treatment at the rate of $50 \mathrm{ppm}$ has the potential to extend the vase life of cut gerbera flowers.

\section{References}

Abdel-Kader H. and Rogers M. N. (1985). The effects of each component of a flower preservative solution on the longevity of cut inflorescences of 3 cultivars of Gerbera jamesonii; ISHS ActaHorticulturae 181(III).

Amerine, M.A., Pangborn, R. M. and Roessler, E. B. (1965). Principle of Sensory Evaluation of Food, Academic Press Inc., New York.

Bieleski RL and Reid MS (1992). Physiological changes accompanying senescence in the ephemeral daylily flower. Plant Physiol., 98:1042-1049.

Chebet, D.K. (1999). Effect of plant growth regulators on the post harvest handling of tuberose (Polianthes tuberosa 1.) cut flowers; Dept of Plant Sci and Crop Protection, University of Nairobi.

Gholami, M., Rahemi, M. and Rastegar, S. (2011). Effect of pulse treatment with sucrose, exogenous benzyl adenine and gibberellic acid on vase life of cut rose 'Red One'Hort, env. And biotech. October 2011, 52(5): 482-487.

Lane, J.H. and Eynon, L. (1923). Determination of reducing sugar by Fehling's solution with methylene blue indicator. J. Soc. Chem. Ind.,42:32.

Lu. P, Cau, J. and He, S. (2010). Nano-silver pulse treatments improve water relations of cut rose cv. Movie Star flowers; Postharvest Boil. and Tech., 57(3): 196-202.

Meeteren, V. (1978). Water relations and keeping-quality of cut Gerbera flowers. I. The cause of stem break; Scientia Horticulturae, 8: 65-74.

Randawa, G.S. and Mukhopadhyay, A. (1986). Floriculture in India: Commercial floriculture. Pub by Sunil Sachdev and printed by Ravi Sachdev at allied Publisher Pvt. Ltd, New Delhi- 110644: 344-425.

Ranganna,S. (2004). Handbook of Analysis and Quality Control for Fruit and Vegetables Products, II $^{\text {nd }}$ edition. Tata and MgGraw- Hill Publishing Co. Ltd., New Delhi.

Rogers, M.N. (1973).A historical and critical review of post harvest physiology research on cut flowers; HortScience, 8: 189-194.

Serek, M., Tamari. G., Sisler, E.C. and Borochov, A. (1995). Inhibition of ethylene-induced cellular senescence symptoms by 1-methylcyclopropene, a 
new inhibitor of ethylene action; Physiol Plant 94:229-232.

Zaky, A., Soad, A.M. Khenizy, and Yasser, M.E. El-Shewaikh. (2013). Effect of
Humic Acid on Vase Life of Gerbera Flowers After Cutting; J. HortlSci\& Ornamental Plants 5(2): 127-136.

\section{How to cite this article:}

Lyang B. Sangma and Chaurasiya, A. K. 2019. Effect of Pulse Treatment on Post Harvest Quality of Gerbera (Gerbera jamesonii Bolus ex Hooker) cv. Black Bird. Int.J.Curr.Microbiol.App.Sci. 8(12): 1380-1392. doi: https://doi.org/10.20546/ijcmas.2019.812.167 